\title{
DIAGNOSTIC ACCURACY OF ELECTRICAL PULP TESTING COMPARED TO THE THERMAL IN PRIMARY TEETH
}

\author{
Dalia M Moheb* and Norhan A El Dokky*
}

\begin{abstract}
Aim: This study aimed to assess the diagnostic accuracy of electric pulp testing compared to thermal method in registering pulp status in primary molars.

Subjects \& Methods: Forty cooperative children with carious primary molars were included in the study. The cold test was carried out using Ethyl Chloride on cotton pellet and was applied to the middle third of the buccal surface until the child indicated that he had felt sensation. The heat test was carried out using heated gutta-percha. Before using the electric pulp tester (EPT) teeth were isolated and dried. The gold standard for pulp status was decided by direct inspection of the presence or absence of bleeding after access cavity had been done. Data were statistically described in terms of frequencies and percentages. Comparison between the different test results was done using McNemar test. Agreement was tested using kappa statistic. Accuracy was represented using the terms sensitivity, specificity, +ve predictive value, -ve predictive value, and accuracy. Comparing accuracy was done using Yates corrected Chi squared test. $p$ values less than 0.05 was considered statistically significant.
\end{abstract}

Results: The highest accuracy was found for EPT followed by heat and cold tests. The highest true negative response was recorded for EPT (47.5\%), then heat and cold (35\%) and (30\%) respectively. Sensitivity for EPT, heat and cold was $84.2 \%, 94.74 \%$ and 84.21 respectively while, specificity was $90.48 \%, 66.67 \%$ and $57.14 \%$.

Conclusions: EPT can be used as a sensible test for diagnosing pulp status in primary teeth.

KEYWORDS: Pulp tester, primary teeth, thermal pulp tester, electrical pulp tester

\section{INTRODUCTION}

Diagnosis is not a goal, but it has been described as a way to treatment decision. Correct diagnosis is the basis for rational therapy and is thus the first step for adequate treatment. Although no single investigation establishes a diagnosis, ignoring relevant investigation may make correct diagnosis impossible ${ }^{(1)}$.

Accurate diagnosis of the pulp status is the most important step to achieve successful endodontic therapy. A combination of the patient's history as well as pulp sensibility tests and radiographic 
data leads the dentist to accurately diagnose the pulp status which then determines the treatment options ${ }^{(2)}$. Frequently, this is overlooked in pediatric patients by clinicians which may result in incorrect decision in treatment planning ${ }^{(3)}$.

There is little information to support that pulp testing of primary teeth is as accurate as permanent teeth ${ }^{(3)}$. Studies have claimed that pulp testing in children below the age of 10 years was considered unreliable because children may not co-operate properly. Moreover, it was suggested that due to the incomplete innervation of newly erupted teeth the results may be affected ${ }^{(4)}$.

Various tests have been used for pulpal diagnosis; pulp testing may involve thermal and electric pulp testing ${ }^{(3)}$.

Thermal tests include cold and heat tests. They work on the principle of hydrodynamic force in dentin. It is supposed that the fluid movement in tubules is responsible for activation of sensory receptors in the pulp ${ }^{(5)}$.

Cold test is done using ice, ethyl chloride, frozen $\mathrm{CO}_{2}$ or a cotton pellet sprayed with difluorodichlormethane ${ }^{(6)}$. Studies have indicated that cold test is more reliable than other thermal tests as it is believed that the colder the stimuli, the more reliable it is to assess the pulp status ${ }^{(1)}$.

Heat test is carried out using hot gutta percha stick, teeth under test should be isolated with cotton, and the stick may be heated till it becomes soft and applied to tooth under investigation. This test may be difficult to carry in posterior teeth due to limited access. Heat test should be done for not more than 5 seconds to avoid damaging the pulp ${ }^{(7)}$.

Electric pulp testing has been introduced and used in dental practices worldwide for more than a century ${ }^{(8)}$. It is based on stimulation of sensory nerves and relies on subjective assessments and comments from the patients. These can lead to falsepositive and false-negative results. Nevertheless,
EPT remains an important aid, and when properly used, it is safe and reliable clinical test that can provide useful information regarding health and disease $^{(9)}$.

Mathews et al, had cast doubt on the ability of the pulp tester to measure responses from the pulp, as there is a risk that responses may result from stimulation of receptors in the supporting tissues ${ }^{(10)}$.

Other researchers concluded that interpretation of a child response to pulp testing is difficult and further complicated because the responses given by the patients are subjective as some children may exaggerate the symptoms due to fear and anxiety ${ }^{(11-12)}$.

Recent recommendations on the benefits of indirect pulp treatment in primary teeth make the importance of pulp diagnosis in pediatric dentistry very crucial ${ }^{(13)}$. Currently, there is quite scarce investigations about the pulp tests in primary teeth even though it is essential for clinicians to evaluate the status of the pulp before deciding a treatment plan $^{(14)}$.

Asfour et al; ${ }^{(15)}$ tested primary canines with an electrical pulp tester and ethyl chloride, they concluded that pulp tester was valid in primary teeth but with no reference to sensitivity, specificity, positive and negative predictive values of these tests.

Hori et al; ${ }^{(16)}$, in another study assessed the ability of thermal and electrical pulp tests to determine the pulp status in primary teeth, they revealed that electric pulp tester was useful for determining the pulp status in primary teeth. The accuracy of electric pulp tester was higher than that of either of the thermal tests as well that of combinations of three diagnostics tests.

To the best of our knowledge, few studies were conducted on primary teeth using EPT. Therefore, the aim of this study was to compare the diagnostic accuracy of different pulp tests in primary teeth. 


\section{SUBJECTS AND METHODS}

This study was a diagnostic accuracy study, it was carried out at Pediatric Dentistry DepartmentFaculty of Dentistry- Cairo University.

The study was carried out in accordance with the ethical guidelines in research with human participants and approved by the Research Ethics Committee of the Faculty of Dentistry, Cairo University (Code 16-1-29). These ethical guidelines are in full accordance with the World Medical Association Declaration of Helsinki.

\section{Eligibility Criteria:}

The children were selected to fulfill the following criteria:

\section{a) Inclusion criteria:}

1) Apparently healthy children with no systemic diseases, neurologic disorders or mental disabilities.

2) Cooperative children (Frankel's ratings 3 and 4).

3) Children with carious primary molars indicated for pulp therapy.

\section{b) Exclusion criteria:}

The presence of periapical or gingival lesion or gingivitis.

The purpose and the procedures were explained to the parent/caregiver and consents were taken from them before intervention. The medical and dental histories of the children were also recorded in patient assessment charts.

Provisional diagnosis of pulp status was carried out through clinical and radiographic examinations before pulp testing of the teeth.

Forty cooperative children (18 males, 22 females) with age range (6-8) years. The children had carious primary molars, indicated for pulp therapy treatment and showing no signs of pathological pulp affection were included in the study, they were selected from
Outpatient clinic of Pediatric Dentistry DepartmentFaculty of Dentistry- Cairo University.

Before starting the testing, each child was instructed to raise his/her hand when he/she felt sensation during the test. All the tests were done on the middle third of the buccal surface of the primary molar that was being tested ${ }^{(16)}$.

All examinations and tests were done by one investigator for standardization purposes.

\section{i. Thermal pulp testing:}

a) The cold test was carried out using Ethyl Chloride (Walter Ritter GmbH, Co- PharmaceuticaGermany) sprayed on size 2 cotton pellet ${ }^{(16)}$, and then applied to the tooth surface and held until the child raised his hand indicating that he/ she had felt sensation.

b) Heat test was carried out using heated guttapercha, before applying the heat test a layer of lubricant was coated on the tooth surface to be tested. Then the heated gutta-percha was applied.

These were applied to the gingival third of the buccal surface of dried and isolated teeth for up to 10 seconds per tooth.

\section{ii. Electric Pulp Testing:}

Before applying the EPT the teeth were isolated with cotton rolls, dried with a piece of gauze. A drop of toothpaste (Signal, Unilever, Egypt) was used as conducting medium. The EPT (Denjoy, Pulp Tester DY 310, Denjoy Dental Co., LTD, China) was used according to manufacturer's instructions.

When finishing each test, an interval time of two minutes was allowed to give chance to the pulp to return to normal condition, before the following test.

The reference point for pulp status was decided by direct inspection of the presence or absence of bleeding in the examined tooth after finishing the access cavity ${ }^{(17-18)}$. 


\section{Statistical analysis:}

Data were statistically described in terms of frequencies and percentages. Comparison between the different test results was done using McNemar test. Agreement was tested using kappa statistic. Accuracy was represented using the terms sensitivity, specificity, +ve predictive value, -ve predictive value, and accuracy. Comparing accuracy was done using Yates corrected Chi squared test.

$p$ - values less than 0.05 was considered statistically significant. All statistical calculations were done using computer program SPSS (Statistical Package for the Social Science; SPSS Inc., Chicago, IL, USA) version 15 for Microsoft Windows.

\section{RESULTS:}

In the current study 40 primary molars were tested. Diagnostic accuracy was studied thoroughly for three pulp testing modalities. The sensitivity, specificity, positive predictive value (PPV), negative predictive value (NPV) were all studied.

The reference point utilized in this study was the clinical appearance of bleeding pulps indicating vitality upon treatment.

The highest true negative (TN) response was recorded for Electric Pulp Tester (EPT) as this was noted in $19(47.5 \%)$ of the studied sample, TN values for heat and cold were 14(35\%) and 12(30\%) respectively as shown in tables $(1,2 \& 3)$.

The highest true positive (TP) response was recorded for Heat Pulp Test as this was noted in $18(45.5 \%)$ of the studied sample, TP values for electric and cold tests were $16(40 \%)$ as shown in tables $(1,2 \& 3)$.

When evaluating the results of Heat Pulp testing, sensitivity which is ability of the test to detect positive response when it really exists was $94.7 \%$. Specificity of the heat test which is the ability of the test to detect negative response when it truly exists was $66.67 \%$.

The positive predictive value (PPV)in case of evaluating the heat test which is the liability of being truly positive if a positive response was recorded by the test was $72 . \%$. The negative predictive value (NPV) which is the liability to be truly negative if the test indicated so was $93.3 \%$.

In order to test the agreement between the test results and the actual state of the pulp Kappa statistics was used. For the heat test the agreement was considered good agreement (0.605).

When the results of cold pulp testing were evaluated sensitivity was $84.21 \%$, specificity was $57.14 \%$. Moreover, PPV was $64 \%$ and NPV was $80 \%$. The agreement with kappa statistics showed poor agreement $(0.407)$.

Finally, upon evaluating the results of EPT, sensitivity was $84.21 \%$ and specificity was $90.48 \%$. While PPV was $88.89 \%$ and NPV was $86.36 \%$. The agreement with kappa statistics showed good agreement (0.749).

The highest accuracy was recorded in electric pulp testing EPT $(87.50 \%)$ followed by heat $(80.00 \%)$ and then cold tests $(70.00 \%)$ as shown in table (4).

The highest sensitivity was reported with heat pulp testing followed by the electric and cold pulp testing whereas the highest specificity was reported in the electric pulp testing followed by the heat and cold pulp testing.

When the sensitivities and specificities of heat and cold test were interrelated, there was no statistical significant difference, as the p-value was (0.597) and (0.751) respectively. Also, sensitivities and specificities of heat and EPT showed no statistical significance difference, with p-value (0.597) and (0.311) respectively. Finally, sensitivities of cold and EPT showed no statistical significant difference 
when the results were interrelated, as p-value was (0.656). However, there was statistical difference when the specificity of electric and cold tests were interrelated with p-value (0.035).

TABLE (1): Diagnostic sensibility of Heat test.

\begin{tabular}{|l|l|l|l|}
\hline Test & \multicolumn{2}{|c|}{ Vital } & \\
\hline Heat & Negative N\% & Positive N\% & Total N\% \\
\hline Negative & $14(35 \%)$ & $1(2.5 \%)$ & $15(37.5 \%)$ \\
\hline Positive & $7(17.5 \%)$ & $18(45 \%)$ & $25(62.5 \%)$ \\
\hline Total & $21(52.5 \%)$ & $19(47.5 \%)$ & $40(100 \%)$ \\
\hline
\end{tabular}

TABLE (2): Diagnostic sensibility of Cold test

\begin{tabular}{|c|c|c|c|}
\hline Test & & Vital & \\
\hline Cold & Negative $\mathbf{N} \%$ & Positive N\% & Total N\% \\
\hline Negative & $12(30 \%)$ & $3(7.5 \%)$ & $15(37.5 \%)$ \\
\hline Positive & $9(22.5 \%)$ & $16(40 \%)$ & $25(62.5 \%)$ \\
\hline Total & $21(52.5)$ & $19(47.5 \%)$ & $40(100 \%)$ \\
\hline
\end{tabular}

TABLE (3): Diagnostic sensibility of Electric test

\begin{tabular}{|l|l|l|l|}
\hline Test & \multicolumn{2}{|c|}{ Vital } & \\
\hline Electric & Negative N\% & Positive N\% & Total N\% \\
\hline Negative & $19(47.5 \%)$ & $3(7.5 \%)$ & $22(55 \%)$ \\
\hline Positive & $2(5 \%)$ & $16(40 \%)$ & $18(45 \%)$ \\
\hline Total & $21(52.5 \%)$ & $19(47.5 \%)$ & $40(100 \%)$ \\
\hline
\end{tabular}

TABLE (4): Diagnostic sensibility results of different tests

\begin{tabular}{|c|c|c|c|c|c|c|c|c|c|}
\hline Test & TP & FN & TN & FP & $\begin{array}{c}\text { Sensitivity } \\
\text { \% }\end{array}$ & $\begin{array}{c}\text { Specificity } \\
\text { \% }\end{array}$ & $\begin{array}{c}\text { (+ve) } \\
\text { PV\% }\end{array}$ & $\begin{array}{c}\text { (-ve) } \\
\text { PV\% }\end{array}$ & $\begin{array}{c}\text { Accuracy } \\
\text { \% }\end{array}$ \\
\hline Heat & 18 & 1 & 14 & 7 & 94.74 & 66.67 & 72.00 & 93.33 & 80.00 \\
\hline Cold & 16 & 3 & 12 & 9 & 84.21 & 57.14 & 64.00 & 80.00 & 70.00 \\
\hline Electric & 16 & 3 & 19 & 2 & 84.21 & 90.48 & 88.89 & 86.36 & 87.50 \\
\hline
\end{tabular}

TP: True Positive

TN : True Negative

FN: False Negative

FP : False Positive

PV: Predictive Value

\section{DISCUSSION}

Accurate assessment of pulp status is one of the greatest diagnostic challenges in clinical practice. Assessment of pulp vitality is not a simple task, especially when dealing with pediatric patients, as their response may not be reliable moreover their anxiety may increase during performing the test ${ }^{(19)}$.

Since only few studies were conducted on primary teeth using EPT. Therefore, this study was conducted aiming to evaluate the diagnostic accuracy of EPT compared to thermal tests as regards the sensitivity, specificity, positive and negative values.

Cold test was carried out using ethyl chloride and heat test using heated gutta percha as they are the most commonly used testing methods ${ }^{(15-20)}$.

On applying the cold test, a size 2 cotton pellet was used because as reported by Jones 1999 it can produce lower temperature than other sizes ${ }^{(16)}$. 
Before applying the EPT the teeth were isolated with cotton rolls to avoid gingival conduction ${ }^{21}$. A drop of toothpaste was used as conducting medium to ensure that maximum current passes from the electrode to the tooth surface ${ }^{(22-23)}$.

In the present study the highest accuracy was found for EPT followed by heat and then cold tests, this was in agreement with Hori et al; 2011(18), while it is in contrast with Petersson et al; ${ }^{(17)}$ who revealed that the highest accuracy was found with cold test followed by the EPT then heat test, this could be due to difference in the tested teeth as the they examined permanent teeth, while in the present study primary teeth were examined.

Results of the present study revealed that the sensitivity of heat test and specificity of EPT were the highest in all sensibility test, this goes in accordance with Hori et al; 2011 ${ }^{(18)}$, this could be attributed to the fact that both studies used similar methods for assessing pulp sensibility regarding diagnostic tests.

Regarding the results of PPV and NPV in the current study, EPT showed the highest PPV, while heat test showed the highest NPV, this is in agreement with Hori et al; $2011^{(18)}$. Since Jafarzadeh and Abbott, $201{ }^{(24)}$ stated that PPV is dependent on specificity while NPV is dependent on sensitivity, then according to the results of the present study EPT is more reliable followed by heat than cold test.

Results of the current study revealed that no significant difference was found between the accuracy of EPT, heat and cold tests ( $p$-value $>0.05$ ), this was in accordance with Asfour et al; $1996{ }^{(15)}$ who reported that there was no significant difference in response rate between EPT and cold test. In contrast to Hori et al; $\mathbf{2 0 1 1}^{(18)}$ who found no significant difference between the accuracy of EPT and the heat test (P-values $>0.05)$; however, the accuracy of EPT was significantly higher than that of the cold test (P-value < 0.05), this may be attributed to different methods of evaluation regarding the model EPT used.

\section{CONCLUSIONS}

1- The accuracy of the EPT was higher than that of heat and cold tests.

2- EPT can be used as a sensible test for diagnosing pulp status in primary teeth.

\section{REFERENCES}

1- Chambers IG. The role and methods of pulp testing in oral diagnosis: a review. IEJ 1982; 15:1-5

2- Chen E, Abbott PV. Dental pulp testing: A review. Int J Dent 2009; doi:10.1155/2009/365785: 1-12.

3- Baume L J. Diagnosis of diseases of the pulp. Oral Surg Oral Med Oral Patho 1970;29: 102-111.

4- Mumford JM. Pain perception threshold and adaptation of normal human teeth. Arch Oral Biol 1965; 10: 957-968.

5- Brannstrom M, Astrom A. The hydrodynamics of the dentin: it's possible relationship to dentinal pain. Int Dent J 1972; 22:219-303

6- Brannstrom M. The hydrodynamic theory of dentinal pain: sensation in preparations, caries and dentinal crack syndrome. J Endod 1986; 12:453- 457.

7- Ehrmann EH. Pulp testers and pulp testing with particular reference to the use of dry ice. Aust Dent J 1977; 22: 272279.

8- Lin J, Chandler NP. Electric pulp testing: A review. Int Endod J 2007; doi:10.1111/j.1365-2591.2008.01375.x: $1-10$.

9- Dummer PMH, Hicks R, Huws D. Clinical signs and symptoms in pulp disease. Int Endod J 1980; 19: 161-171.

10- Mathews B, Searle EN, Adams D , Linden R. Thresholds of vital and non-vital teeth to stimulation with electric pulp testers. Br Dent J 1974; 137: 352-355.

11- Gaffney A, Dunne EA. Developmental aspects of children's definition of pain. Pain 1986; 26: 105-1 17

12- Eli I. Dental anxiety: A cause for possible misdiagnosis of tooth vitality. Int Endod J 1993; 26: 251-253.

13- Fuks AB. Vital pulp therapy with new materials for primary teeth: new directions and treatment prospective. J Endod 2008; 34(Suppl.): S18-S24.

14- Gopikrishna V, Pradeep G, Venkateshbabu N. Assessment of pulp vitality: a review. Int J Paediat Dent 2009; 19: 3-15. 
15- Asfour MA, Miller BJ, Smith PB. An assessment of the reliability of pulp testing in deciduous teeth. Int J Paediat Dent 1996; 6: 163-166.

16- Jones DM. Effect of the type carrier used in the results of dichlorodifluoromethane application to teeth. J Endod 1999; 25: 692-694.

17- Petersson K, Soderstrom C, Kiani Anaraki M, Levy G. Evaluation of the ability of thermal and electrical tests to register pulp vitality. Endod Dent Traumat1999; 15: 127131.

18- Hori A, Poureslami HR, Parirokh M, Mirzazadeh A, Abbott P. The ability of pulp sensibility tests to evaluate the pulp status in primary teeth. Int J Pediatr Den 2011; 21: 441-445.
19- Diwanji A, Mathur J, Sharma D, Akhani J. Assessment of pulp vitality in children. J Dent Scienc 2012; 2(2): 44-46.

20- Davies AL, Rawlinson A. A comparison between two electric vitality testers and ethyl chloride with special reference to a newly available device. Int J Endod 1988; 21: $320-326$.

21- Myers JW. Demonstration of a possible source of error with an electric pulp tester. J Endod 1998; 24: 199-200.

22- Michaelson RE, Seidberg BH, Guttuso J. An in vivo evaluation of interface media used with the electric pup tester. JADA 1975; 91: 118-121.

23- Cooley RL, Robison SF. Variables associated with electric pup testing. Oral Surg, Oral Med, Oral Pathol 1984; 58:437-442. 